\title{
Strong enhancement of magnetic dipole emission in a multilevel electronic system
}

\author{
Sinan Karaveli and Rashid Zia* \\ School of Engineering, Brown University, Providence, Rhode Island 02912, USA \\ *Corresponding author: Rashid_Zia@brown.edu
}

Received June 24, 2010; revised August 17, 2010; accepted August 26, 2010;

posted September 17, 2010 (Doc. ID 130673); published October 8, 2010

\begin{abstract}
The Purcell effect is commonly used to increase light emission by enhancing the radiative decay of electric dipole transitions. In this Letter, we demonstrate that the opposite effect, namely, the inhibition of electric dipole transitions, can be used to strongly enhance light emission via magnetic dipole transitions. Specifically, by exploiting the differing symmetries of competitive electric and magnetic dipole transitions in trivalent europium, we demonstrate a fourfold enhancement of the far-field emission from the ${ }^{5} D_{0} \rightarrow{ }^{7} F_{1}$ magnetic dipole transition in trivalent europium. We show that this strong enhancement is well predicted by a three-level model that couples the individual Purcell enhancement factors of competitive transitions from the same excited state. (c) 2010 Optical Society of America

OCIS codes: $\quad 160.5690,300.2140,310.6188$.
\end{abstract}

Engineering the radiative decay of excited emitters is a critical step in the design of many optoelectronic devices, including lasers, LEDs, and optical amplifiers. In this context, the Purcell effect can be used to either enhance or inhibit spontaneous decay by modifying the accessible electromagnetic modes in an emitter's local environment [1]. Although Purcell's original work described the decay of nuclear magnetic resonances, this effect has become synonymous with enhancing light emission from electric dipole (ED) transitions. Numerous studies have demonstrated enhanced emission from the ED transitions of semiconductor quantum dots within optical microcavities and photonic crystals [2]. Given the prevalence of ED transitions over higher-order processes (e.g., magnetic dipole, electric quadrupole), the focus on enhancing ED transitions is easily understood. In this Letter, however, we investigate how inhibiting a dominant ED transition can produce an increase in observed emission from magnetic dipole (MD) transitions in multilevel electronic systems.

Interest in optical frequency magnetic resonances has been reignited by pioneering research in the field of metamaterials. Tremendous progress has been made by engineering magnetic resonances in metal nanostructures, such as split ring resonators and paired nanorods [3], and this booming field has helped renew interest in the natural MD transitions of lanthanide ions. In 2006, Thommen and Mandel proposed that the degenerate $\mathrm{ED}$ and $\mathrm{MD}$ transitions of trivalent erbium $\left(\mathrm{Er}^{3+}\right)$ could be used to produce negative-index response at telecommunication frequencies [4] and, more recently, Noginova et al. proposed that MD transitions of trivalent europium $\left(\mathrm{Eu}^{3+}\right)$ could be used as local probes of optical frequency magnetic fields $[5,6]$. These and other lanthanide ions exhibit strong MD transitions that are ubiquitous light emitters in applications ranging from fluorescent lighting to erbium-doped fiber amplifiers. For example, MD emission can account for up to $35 \%$ of the light emitted by $\mathrm{Er}^{3+}$ at the ${ }^{4} I_{13 / 2} \rightarrow{ }^{4} I_{15 / 2}$ transition around $1.55 \mu \mathrm{m}$ [6]. Consequently, enhancing the MD transitions of lanthanide ions could have important applications in optoelectronic devices and metamaterials research.
To date, the Purcell effect has mostly been studied in the context of two-level ED emitters. Even $\mathrm{Er}^{3+}$, which has a multitude of energy levels and transitions, is often effectively modeled as a two-level ED emitter for analyzing Purcell enhancements [7,8]. Here, we show that competing transitions in multilevel lanthanide ions can be used to strongly enhance MD emission. Specifically, we demonstrate that the differing symmetries and spectral proximity of ${ }^{5} D_{0} \rightarrow{ }^{7} F_{1}$ MD and ${ }^{5} D_{0} \rightarrow{ }^{7} F_{2}$ ED transitions in $\mathrm{Eu}^{3+}$ can be leveraged to get more emission via the MD transition. Furthermore, we show that our experimental results are in agreement with a three-level model for $\mathrm{Eu}^{3+}$ that couples the Purcell factors for these competing transitions.

The emission spectrum of $\mathrm{Eu}^{3+}$ exhibits several $\mathrm{ED}$ transitions from the ${ }^{5} D_{0}$ excited state to ${ }^{7} F_{0},{ }^{7} F_{2},{ }^{7} F_{3}$, and ${ }^{7} F_{4}$, as well as an MD transition from ${ }^{5} D_{0}$ to ${ }^{7} F_{1}$ [9]. All of these transitions are coupled to each other via the ${ }^{5} D_{0}$ excited state. Consequently, inhibition of one transition enhances the probability of decay via others. A model system with which to investigate such

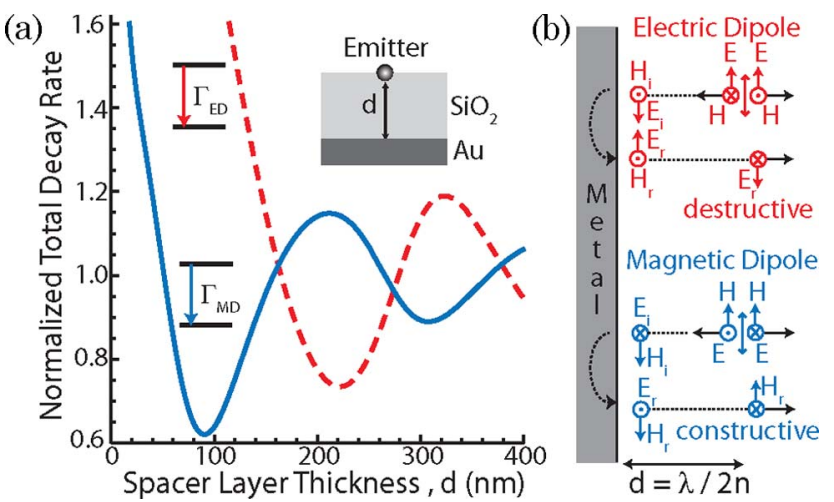

Fig. 1. (Color online) (a) Normalized total radiative decay rates of two-level magnetic (solid curve, $\lambda=590 \mathrm{~nm}$ ) and electric (dashed curve, $\lambda=610 \mathrm{~nm}$ ) dipole emitters as a function of distance from a planar gold surface. $\varepsilon_{\mathrm{SiO}_{2}}=2.146$ and $\varepsilon_{\mathrm{Au}}$ from [13]. Inset, schematic of calculation geometry. (b) Illustrative example showing differing self-interference for tangentially oriented ED and MD emitters located a half-wavelength from a metal surface. 
coupling is an emitter near a reflective, planar metal surface. Depending on its distance from the metal surface, the emitter's radiative decay rate will be modified by the Purcell effect [10]. This modification is well described by classical self-interference with reflected fields at the emitter's location. The modified decay rate for a twolevel ED emitter with unit quantum efficiency embedded at the air-exposed surface of a glass layer of thickness $d$ on top of a gold mirror [see inset Fig. 1(a)] is given by [11]

$$
\begin{aligned}
\Gamma_{\perp}^{\mathrm{ED}}(d)= & \Gamma_{0}^{\mathrm{ED}}\left(\frac{3}{2} \mathrm{Im}\right. \\
& \left.\times\left[\int_{0}^{\infty} \frac{\left(1-r_{12}^{p} \exp (-2 l k d)\right)\left(1+r_{13}^{p}\right)}{1-r_{12}^{p} r_{13}^{p} \exp (-2 l k d)} \frac{u^{3} \mathrm{~d} u}{l}\right]\right), \\
\Gamma_{\|}^{\mathrm{ED}}(d)= & \Gamma_{0}^{\mathrm{ED}}\left(\frac { 3 } { 4 } \operatorname { I m } \left[\int _ { 0 } ^ { \infty } \left(\left(1-u^{2}\right)\right.\right.\right. \\
& \times \frac{\left(1+r_{12}^{p} \exp (-2 l k d)\right)\left(1+r_{13}^{p}\right)}{1-r_{12}^{p} r_{13}^{p} \exp (-2 l k d)} \\
& \left.\left.\left.+\frac{\left(1+r_{12}^{s} \exp (-2 l k d)\right)\left(1+r_{13}^{s}\right)}{1-r_{12}^{s} r_{13}^{s} \exp (-2 l k d)}\right) \frac{u \mathrm{~d} u}{l}\right]\right),
\end{aligned}
$$

where $\Gamma_{\perp}^{\mathrm{ED}}$ and $\Gamma^{\mathrm{ED}}$ are the total decay rates for an ED oriented perpendicular and parallel to the interface and $\Gamma_{0}^{\mathrm{ED}}$ is the total decay rate in a homogeneous glass sample. $r_{i, j}^{s}$ and $r_{i, j}^{p}$ are the reflection coefficients for $s$ - and $p$ polarized plane waves from the $i, j$ interface, with indices 1,2 , and 3 designating glass, gold, and air media, respectively. $u$ and $l$ are the parallel and perpendicular normalized components of the wave vector $\mathbf{k}$ in glass given by $u=k_{\|} / k$ and $l=-i \sqrt{1-u^{2}}$. Equation (1) can also effectively model two-level MD emitters by interchanging $r_{i, j}^{s}$ with $-r_{i, j}^{p}[\underline{11}]$. These expressions for total decay rate include both far-field $(0 \leq u<1)$ and near-field $(u>1)$ radiation components. For comparison with ensemble experiments, these rates are averaged over dipole orientation $\left(\Gamma_{\text {iso }}=1 / 3 \Gamma_{\perp}+2 / 3 \Gamma_{\|}\right)$and normalized to a semi-infinite glass reference case without gold, i.e., $\gamma=\Gamma_{\text {iso }} / \Gamma_{\text {iso }}^{\text {ref }}$

Figure 1(a) shows $\gamma$ as a function of glass layer thickness $d$ for an ED emitting at $610 \mathrm{~nm}$ and an MD emitting at $590 \mathrm{~nm}$. The figure shows several features of interest. First, the emitter-metal distance determines the phase of the reflected field at the point of the emitter; when in phase (out of phase) with the field propagating away from the metal interface, emission is enhanced (inhibited). Second, it shows that, for the same separation distance, MD and ED decay rates are inversely related; when one is enhanced, the other is generally suppressed. This trend results from the differing field symmetries for ED and MD emitters [12]. As shown in Fig. 1(b), the electric (magnetic) field radiated by an ED (MD) emitter is symmetric about the dipole axis, whereas the magnetic (electric) field is antisymmetric. Near an interface, the $\pi$-phase difference between reflected electric and magnetic fields results in destructive interference of an ED emitter at the same distance for which an MD emitter experiences constructive interference. Previous research on $\mathrm{Eu}^{3+}$ has mainly focused on demonstrating this symmetry difference without an emphasis on enhancing MD emission [10-12]. Given the competitive nature of ED and $\mathrm{MD}$ transitions in $\mathrm{Eu}^{3+}$, however, their differing symmetries can also be exploited to strongly enhance MD emission by inhibiting the dominant ED transition.

To demonstrate this effect, we fabricated the structure shown in the inset of Fig. 2(a). Starting with glass coverslips, electron-beam evaporation was used to deposit a $10 \mathrm{~nm} \mathrm{Ti}$ adhesion layer and a $100 \mathrm{~nm}$ Au film. Subsequently, different thickness $\mathrm{SiO}_{2}$ layers were deposited using plasma-enhanced chemical vapor deposition. The thickness of each layer was confirmed by ellipsometry. Finally, a $10 \mathrm{~nm}$ layer of tris(dibenzoylmethane) mono (1,10-phenanthroline)-Eu ${ }^{3+}, \quad \mathrm{Eu}(\mathrm{DBM})_{3}$ phen, (SigmaAldrich 538965) was thermally evaporated on all samples simultaneously, including a pure glass reference sample. Concurrent evaporation helped to ensure a consistent emitter layer thickness for all studied samples.

Emission spectra of $\mathrm{Eu}(\mathrm{DBM})_{3}$ phen for several representative spacer layer thicknesses are shown in Fig. 2(a). Samples were excited with a $337 \mathrm{~nm}$ pulsed $\mathrm{N}_{2}$ laser (SRS NL100), and emission was collected in an inverted microscope with a grating spectrometer (Acton SP2300i). Radiative transitions from the ${ }^{5} D_{0}$ excited state to the ${ }^{7} F_{J}$ ground state manifolds were observed for $J=0$ to 4 . Two transitions of interest for our study are the dominant ${ }^{5} D_{0} \rightarrow{ }^{7} F_{2}$ ED transition near $610 \mathrm{~nm}$ and the ${ }^{5} D_{0} \rightarrow{ }^{7} F_{1}$ MD transition near $590 \mathrm{~nm}$. Figure 2(a) shows asacquired data after background subtraction for dark counts and readout noise. The spectra confirm two
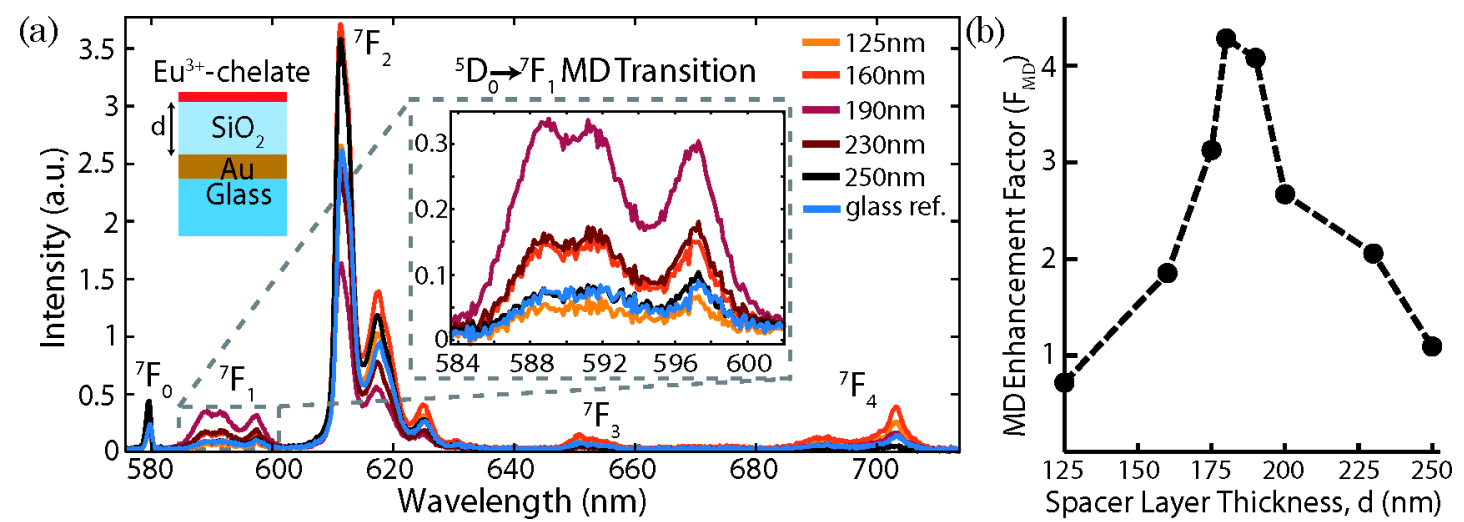

Fig. 2. (a) Representative emission spectra of $\mathrm{Eu}(\mathrm{DBM})_{3}$ phen thin films showing strong modification of emission for different spacer layer thicknesses, $d$, above gold film. Left inset, schematic of fabricated structure. Right inset, magnified spectra of MD emission. (b) MD enhancement factor as a function of spacer layer thickness. 

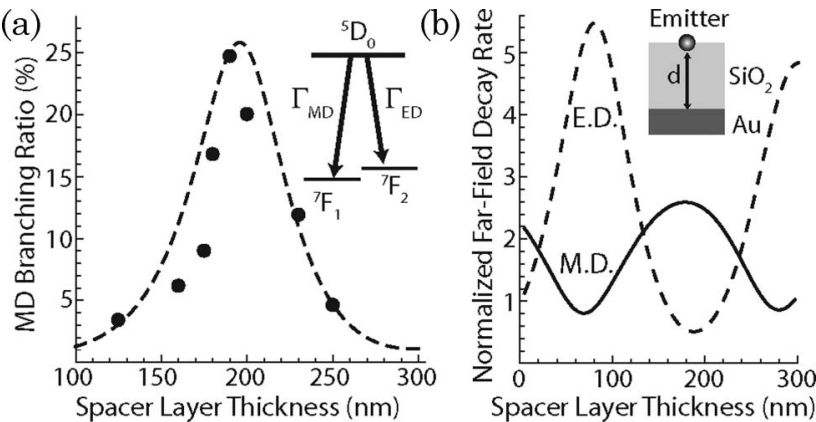

Fig. 3. (a) Comparison of experimental (dots) and calculated (dashed curve) MD branching ratios as a function of spacer layer thickness. Inset, three-level model used for theoretical analysis. (b) Normalized far-field decay rates for isotropic two-level ED (dashed curve, $\lambda=610 \mathrm{~nm}$ ) and MD (solid curve, $\lambda=590 \mathrm{~nm}$ ) emitters. Rates are normalized to the reference case of emitter on pure glass substrate.

theoretical predictions: (1) the MD is enhanced for an $\sim \lambda / 2 n$ spacer layer thickness of around $190 \mathrm{~nm}$, and (2) the dominant $\mathrm{ED}$ is inhibited when the $\mathrm{MD}$ is enhanced. In addition, there is a significant increase of MD emission as compared with the reference $\mathrm{Eu}(\mathrm{DBM})_{3}$ phen on a glass coverslip without any reflecting metal surface [see blue curve in Fig. 2(a)].

The Purcell enhancement for a two-level emitter is commonly quantified by the increase of its total decay rate, $\Gamma / \Gamma_{0}$, which can be inferred from the emitter's lifetime. However, in the case of $\mathrm{Eu}^{3+}$, several radiative transitions originate from the same excited state, ${ }^{5} D_{0}$. Because emission is dominated by the ${ }^{7} F_{2}$ ED transition, the measured lifetime is generally dominated by its changes, obscuring the effect of rate modifications to the ${ }^{7} F_{1}$ MD transition. Therefore, when quantifying changes to weak transitions in a multilevel emitter, it is helpful to look at light intensity modifications. Accordingly, we define both an MD enhancement factor by comparing the MD emission intensity with that of the reference sample, $F_{\mathrm{MD}}=I_{\mathrm{MD}} / I_{\mathrm{MD}}^{\mathrm{ref}}$, where $I_{\mathrm{MD}}=\int_{585}^{601} I(\lambda) \mathrm{d} \lambda$, and an apparent branching ratio for the MD transition defined as $\beta_{\mathrm{MD}}=I_{\mathrm{MD}} / I_{\text {total }}$, where $I_{\text {total }}=\int_{578}^{710} I(\lambda) \mathrm{d} \lambda$, for each spacer layer thickness. These experimental enhancement factors and branching ratios are plotted in Figs. 2(b) and 3(a), respectively. For separation distances of 180 and $190 \mathrm{~nm}$, where self-interference should enhance MD decay and inhibit ED decay, we observe a large fourfold enhancement in MD emission intensity and a fivefold increase in $\beta_{\mathrm{MD}}$ as compared to the glass reference sample $\left(\beta_{\mathrm{MD}}^{\mathrm{ref}}=5.2 \%\right)$. These enhancements are significantly larger than might be inferred from the modest $15 \% \mathrm{MD}$ total decay rate enhancement predicted by the two-level calculation shown in Fig. 1(a).

To accurately predict the observed enhancement, one must account for two important effects. First, the selfinterference phenomenon modifying the total decay rate of a dipole emitter (as shown in Fig. 1) becomes more pronounced when considering only far-field radiation. Figure $3(\mathrm{~b})$ shows the normalized radiative decay rate enhancement into the far-field modes collected by our optical system $\left(\gamma_{\text {far-field }}=\Gamma_{\text {far-field }} / \Gamma_{\text {far-field }}^{\text {ref }}\right)$. We calculate $\Gamma_{\text {far-field }}$ by limiting the upper bound of the integrands in Eq. (1) to the NA of the collection objective $\left(u_{\max }=0.85\right)$, and normalize to $\Gamma_{\text {far-field }}^{\text {ref }}$ for the air-glass reference case using Eqs. 2.37-2.40 in [11]. Note that the differing mean values for ED and MD emitters in this plot are a result of their different collection efficiencies. Second, we must take into account the competitive nature of ED and MD transitions from the ${ }^{5} D_{0}$ excited state. For simplicity, we model $\mathrm{Eu}^{3+}$ as a three-level emitter having a quantum yield of 1 with a dominant $\mathrm{ED}\left({ }^{7} F_{2}\right)$ and an MD $\left({ }^{7} F_{1}\right)$ transition. Theoretically, the observed MD branching ratio is equal to the ratio of radiative decay rates into the collected far-field modes: $\beta_{\mathrm{MD}}=\Gamma_{\text {far-field }}^{\mathrm{MD}} / \Gamma_{\text {far-field }}^{\text {total }}$. Since total emission is dominated by the ED transition, we can approximate $\beta_{\mathrm{MD}} \approx \Gamma_{\text {far-field }}^{\mathrm{MD}} / \Gamma_{\text {far-field }}^{\mathrm{ED}}$. To compare our experimental results with the calculated decay rates shown in Fig. 3(b), we expand $\beta_{\mathrm{MD}}$ in terms of the branching ratio for the glass reference sample as $\beta_{\mathrm{MD}}(d) \approx \beta_{\mathrm{MD}}$ (ref) $\gamma_{\text {far-field }}^{\mathrm{MD}}(d) / \gamma_{\text {far-field }}^{\mathrm{ED}}(d)$. This shows that $\beta_{\mathrm{MD}}$ is increased both by enhancement of the weak MD transition and by suppression of the dominant ED transition. Figure 3(a) shows the strong agreement between the experimental and predicted $\beta_{\mathrm{MD}}$ as a function of spacer layer thickness.

In conclusion, by placing $\mathrm{Eu}^{3+}$ emitters close to a reflective planar metal film, we have shown a fourfold increase of MD light emission intensity. The observed enhancement leverages the competitive nature of the dominant ${ }^{5} D_{0} \rightarrow{ }^{7} F_{2}$ ED and ${ }^{5} D_{0} \rightarrow{ }^{7} F_{1}$ MD transitions and their opposing symmetries. The good agreement between theory and experiment highlights the importance of multilevel electronic structure for the Purcell enhancement of lanthanide emitters. These results, obtained with the spectrally distinct $\mathrm{ED}$ and $\mathrm{MD}$ transitions of $\mathrm{Eu}^{3+}$, also demonstrate a simple method by which to tune the emission spectra of this important lighting phosphor. In the future, similar techniques could be explored to modify the polarization of emission from degenerate $\mathrm{ED}$ and MD transitions, such as the $1.55 \mu \mathrm{m}$ line in trivalent erbium.

This research was supported by a U.S. Air Force Office of Scientific Research (USAFOSR) PECASE award (FA-9550-10-1-0026) and a National Science Foundation (NSF) CAREER award (EECS-0846466).

\section{References}

1. E. M. Purcell, Phys. Rev. 69, 681 (1946).

2. K. J. Vahala, Nature 424, 839 (2003).

3. V. M. Shalaev, Nat. Photon. 1, 41 (2007).

4. Q. Thommen and P. Mandel, Opt. Lett. 31, 1803 (2006).

5. N. Noginova, G. Zhu, M. Mavy, and M. A. Noginov, J. Appl. Phys. 103, 07E901 (2008).

6. N. Noginova, Y. Barnakov, H. Li, and M. Noginov, Opt. Express 17, 10767 (2009).

7. E. Snoeks, A. Lagendijk, and A. Polman, Phys. Rev. Lett. 74, 2459 (1995).

8. Y. Gong, S. Yerci, R. Li, L. Dal Negro, and J. Vuckovic, Opt. Express 17, 20642 (2009).

9. S. Freed and S. I. Weissman, Phys. Rev. 60, 440 (1941).

10. K. H. Drexhage, Prog. Opt. 12, 162 (1974).

11. R. R. Chance, A. Prock, and R. Silbey, Adv. Chem. Phys. 37, 1 (1978).

12. R. E. Kunz and W. Lukosz, Phys. Rev. B 21, 4814 (1980).

13. A. D. Rakic, A. B. Djurišic, J. M. Elazar, and M. L. Majewski, Appl. Opt. 37, 5271 (1998). 